\title{
Clarificación de aguas residuales de la industria láctea en base a la tuna (Opuntia ficus indica) como coagulante natural
}

\begin{abstract}
(c)
\end{abstract}
Clarification of wastewater from the dairy industry based on prickly pear (Opuntia ficus indica) as a natural coagulant

\begin{abstract}
Danielita Fernanda Borja Mayorga. ${ }^{1}$, Marco Raul Chuiza Rojas. ${ }^{2}$ \& Mónica Lilian Andrade Avalos. ${ }^{3}$
\end{abstract}

\begin{abstract}
.
DOI: https://doi.org/10.33262/concienciadigital.v4i3.1.1821

Introduction. The use of natural coagulants emerges as an ecological alternative for both domestic and industrial wastewater treatment. Objetives. The present work investigates the clarification of a wastewater from a dairy industry, in the province of Pastaza, Ecuador, using prickly pear cactus (Opuntia ficus indica) as a natural coagulant agent. Methodology. It was studied the coagulation in samples of one litre of wastewater through a standard jar testing equipment, modifying the coagulant concentration (1-2 $\mathrm{w} \%)$ and volume of coagulant $(5-35 \mathrm{ml})$. The parameters analyzed were turbidity, color, chemical oxygen demand (COD), biological oxygen demand (BOD), suspended solids (SS), total solids (ST) and fecal coliforms (FC), data compared with the Unified Text regulations. of Secondary Environmental Legislation of the Ministry of the Environment (TULSMA). Results. It was found that the optimal

\footnotetext{
1 Facultad de Ingeniería Química, Escuela Politécnica Superior de Chimborazo, Riobamba-Ecuador, dborja@espoch.edu.ec, https://orcid.org/0000-0002-8438-064X

2 Facultad de Ingeniería Química, Escuela Politécnica Superior de Chimborazo, Riobamba-Ecuador, m.chuiza@espoch.edu.ec, https://orcid.org/0000-0003-1908-8033

3 Facultad de Ingeniería Química, Escuela Politécnica Superior de Chimborazo, Riobamba-Ecuador, moandrade@espoch.edu.ec, https://orcid.org/0000-0001-5736-5607
} 
dosage was $20 \mathrm{ml}$ of coagulant solution at $2 \%(400 \mathrm{ppm})$. The removal efficiencies found were $77.8 \%$ in turbidity, $51.8 \%$ in color, $26.8 \%$ in COD, $13.9 \%$ in BOD, $31.0 \%$ in SS, $26.4 \%$ in ST and $99.96 \%$ in CF. Conclusion. The results show that the prickly pear cactus (Opuntia ficus indica) can be used as pretreatment of wastewater from a dairy industry.

Keywords: Clarification/ Wastewater treatment/ Opuntia ficus indica / Coagulatión/ dairy industry.

\section{Resumen.}

Introducción. La utilización de coagulantes naturales surge como una alternativa ecológica para el tratamiento de aguas residuales, tanto domésticas como industriales. Objetivo. Investigar el proceso de clarificación de un agua residual de una industria láctea, de la provincia de Pastaza, Ecuador, utilizando Tuna (Opuntia ficus indica) como agente coagulante natural. Metodología. Se estudió la coagulación en muestras de un litro de agua residual mediante un equipo estándar de prueba de jarras, modificando las variables concentración de coagulante (1-2 \% $\mathrm{P}$ ) y volumen de coagulante $(5-35 \mathrm{ml})$. Los parámetros analizados fueron turbidez, color, demanda química de oxígeno (DQO), demanda bioquímica de oxígeno (DBO), sólidos suspendidos (SS), sólidos totales (ST) y coliformes fecales (CF), datos comparados con la normativa del Texto Unificado de Legislación Ambiental Secundaria del Ministerio del Ambiente (TULSMA). Resultados. Se encontró que la dosificación óptima fue $20 \mathrm{ml}$ de solución de coagulante al 2\% (400 ppm). Las eficiencias de remoción encontradas fueron 77,8\% en turbidez, $51,8 \%$ en color, $26,8 \%$ en DQO, 13,9\% en DBO, 31,0\% en SS, 26,4\% en ST y $99,96 \%$ en CF. Conclusión. Los resultados muestran que la Tuna (Opuntia ficus indica) puede ser utilizada como tratamiento preliminar de las aguas residuales de una industria láctea.

Palabras claves: Clarificación/Tratamiento de aguas residuales/ Opuntia ficus indica / Coagulación/Industria láctea.

\section{Introducción.}

La industria láctea se caracteriza por su elevado consumo de agua y por lo tanto genera gran cantidad de aguas residuales ( 0,2 a 10 litros de efluentes por litro de leche procesada) (Vourch y col., 2008). El agua se usa en las distintas etapas del procesamiento de la leche las cuales incluyen la limpieza, la desinfección, el calentamiento, el enfriamiento y el lavado de pisos. Los mayores contaminantes son la demanda biológica de oxígeno (DBO), la demanda química de oxígeno (DQO), los sólidos suspendidos (SS) y los sólidos disueltos (ST) (Buntner y col., 2013).

Se han utilizado diferentes tecnologías para el tratamiento de las aguas residuales de la industria láctea, entre los cuales se pueden mencionar los tratamientos con membranas (Luo y col., 2011, Vourch y col., 2008, Sarkar y col., 2006); los 
tratamientos biológicos aerobios y anaerobios (Karadag y col., 2015, Buntner y col., 2013, Kaewsuk y col., 2010); los electrotratamientos (Prakash y col., 2011, Yavuz y col., 2011); adsorción con Chitosan y carbón activado (Selmer y col., 1996, Geetha y col., 2012, Prakash y col., 2010) y coagulación-floculación (Prakash y col., 2010, Formentini y col., 2013).

El pretratamiento de las aguas de la industria láctea mediante coagulación ha sido investigado por diferentes autores. El uso de Sulfato de aluminio, Cloruro férrico y Cloruro de polialuminio a concentraciones de $800 \mathrm{mg} / \mathrm{l}$ demostró ser el valor de mayor eficiencia para la remoción de la turbidez de un agua residual de una industria láctea en China (Chen y col., 2012). La eficiencia de remoción de turbidez se reportó por encima de $80 \%$. El coagulante más eficiente fue el Cloruro de polialuminio.

Aunque el uso de coagulantes de sales metálicas resulta más eficiente y económico, se presentan dudas por el efecto del aluminio residual y la enfermedad de Alzheimer. Así pues, el uso de coagulantes naturales surge como una alternativa de tratamiento (Diaz y col., 1999). El Chitosan, sustancia que se obtiene a partir de conchas de camarón se ha utilizado como coagulante para la remoción de color y COD en aguas residuales de la industria láctea (Selmer y col., 1996, Geetha y col., 2012) donde se obtuvieron remociones de COD por encima de 50\%. Adicionalmente, se han hecho experimentos con Moringa Oleífera donde se lograron remociones de más de $93 \%$ en color y turbidez, y $39 \%$ en COD a un agua residual láctea (Fromentini y col., 2013). También se puede mencionar el uso de Cactus latifaria y semillas de Prosopis juliflora (Diaz y col., 1996) donde se obtuvieron remociones mayores a $90 \%$ en turbidez para un agua residual sintética con rangos de turbidez inicial entre 20-200 NTU.

La Tuna (Opuntia ficus indica) pertenece a la familia Cactaceae y crece en climas áridosy semiáridos de Latinoamérica, sur África y países mediterráneos. Se ha encontrado que posee propiedades antioxidantes, antibacterianas y antiinflamatorias y además contiene ácido ascórbico, vitamina E, carotenoides, aminoácidos y antioxidantes (fenoles, flavonoides, betaxantina y betacianina) (El-Mostafa y col., 2014, Lozoya y col., 2018, Gade y col., 2010). Adicionalmente, se ha usado como agente coagulante en el tratamiento de aguas residuales, como por ejemplo en aguas de la industria de pinturas base agua (Vishali y col., 2014); en industrias de pigmentos y textiles (Barka y col., 2013, Louati y col., 2018, Pelaez y col., 2013); en aguas de producción de petróleo (Choudhary y col., 2019, Wan y col., 2019); en combinación con electrotratamientos para la eliminación de turbidez (Djerroud y col., 2018); en aguas residuales de tenerías (Gomes y col., 2015) y en remoción de plomo y cadmio (Onditi y col., 2016). No se encontraron publicaciones con su uso para el tratamiento de aguas de la industria láctea.

El constituyente principal de la Tuna es un heteropolisacarido con un peso molecular de 2,3-300 $\times 10^{4} \mathrm{~g} / \mathrm{gmol}$ que es el responsable de las propiedades coagulantes (Vishali y col., 2014).

El presente trabajo tiene por objetivo estudiar el poder como coagulante de la Tuna (Opuntia ficus indica) en términos de la remoción de color y turbidez de un agua 
residual de una industria láctea ecuatoriana. También se determinarán la eficiencia en remoción de DBO, DQO, SS, ST y coliformes fecales.

\section{Metodologia.}

\section{Material y Métodos}

\section{Agua Residual}

Para la presente investigación se tomaron aproximadamente 80 litros del agua residual de la industria láctea Virgen del Cisne, ubicada en el km 16 de la vía Puyo- Macas, de la Provincia de Pastaza, Ecuador, dedicada a la elaboración de quesos pasteurizados y mozarela. En la Tabla 1 se muestra la caracterización del agua residual usada.

TABLA 1: Caracterización del agua residual usada

\begin{tabular}{lcccc}
\hline Parámetro & Unidades & Método* $^{*}$ & Valor & TULSMA \\
\hline pH & Und. & $4500-\mathrm{B}$ & 418 & $6-9$ \\
Conductividad & $\mu$ Siems/cm & $2510-\mathrm{B}$ & 1,060 & - \\
Color & Und. Co/Pt & - & 3,900 & - \\
Turbiedad & $\mathrm{NTU}$ & $2130-\mathrm{B}$ & 361 & - \\
DBO & $\mathrm{mg} / \mathrm{L}$ & $5220-\mathrm{C}$ & 4,915 & 200 \\
DQO & $\mathrm{mg} / \mathrm{L}$ & $5210-\mathrm{B}$ & 2,895 & 100 \\
SS & $\mathrm{mg} / \mathrm{L}$ & $2540-\mathrm{D}$ & 1,525 & 130 \\
ST & $\mathrm{mg} / \mathrm{L}$ & $2540-\mathrm{A}$ & 7,332 & 1600 \\
CF & $\mathrm{UFC} / 100$ & Microfiltr & $2,4 \times 10^{6}$ & 2000 \\
& $\mathrm{~mL}$ & & \\
\hline Fuente: Grupo Investigación Facultad Ciencias, 2018
\end{tabular}

\section{Coagulante}

\section{Caracterización de la materia prima}

El coagulante utilizado fue la Tuna (Opuntia ficus indica). Para la caracterización se utilizó una penca de Tuna a la cual se le determinaron los siguientes parámetros: $\mathrm{pH}$, humedad, hierro y calcio. La humedad se determinó por gravimetría y los metales con un equipo de absorción atómica VARIAN SpectrAA 55B.

\section{Obtención del coagulante natural}

En la Gráfico 1 se muestra el procedimiento para la obtención del coagulante a partir de las pencas de Tuna. 


\section{GRÁFICO 1. Procedimiento para la obtención de coagulante}

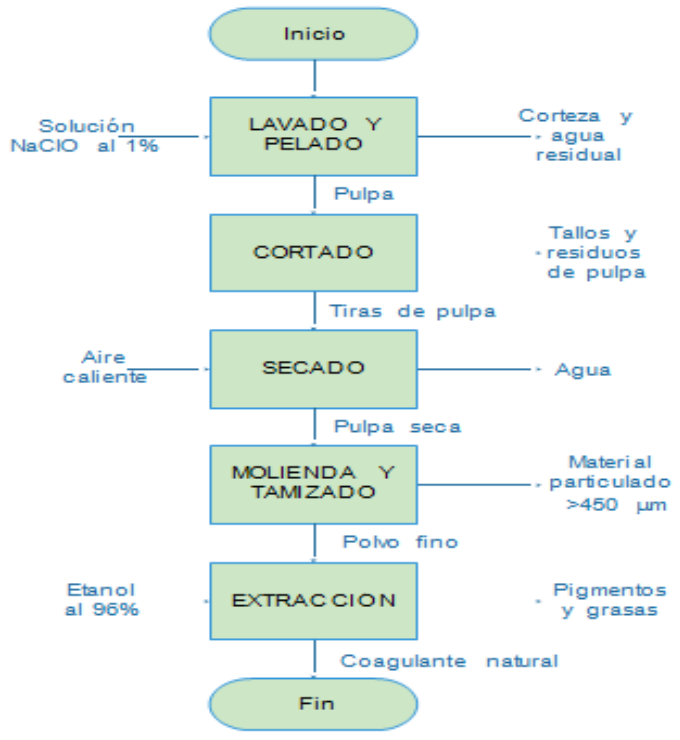

Fuente: Grupo Investigación Facultad Ciencias, 2018

El lavado se realizó con una solución al $1 \%$ de $\mathrm{NaClO}$ para eliminar las impurezas presentes en las pencas de la planta. Luego se procedió a separar las cutículas manualmente con un cuchillo. El cortado se realiza en tiras para aumentar el área de transferencia de calor. El secado se llevó a cabo en una estufa a $60^{\circ} \mathrm{C}$ por 48 horas. Para la molienda se utilizó un molino de tornillo sin fin durante 30 minutos. Para el tamizado de la pulpa molida se usó un tamiz de luz de malla de $450 \mu \mathrm{m}$. El proceso de extracción se realizó con etanol al $96 \%$ por un periodo de 7 horas en un equipo Soxhlet para extraer los pigmentos. Finalmente, se secó el polvo para eliminar el etanol residual, en una estufa a $60^{\circ}$ por dos horas. En cada fase se realizó un balance de masa para estimar el rendimiento por etapa y el global.

\section{Composición del coagulante natura}

Se determinaron los porcentajes de humedad, cenizas, fibra, grasa, proteína y carbohidratos totales del coagulante natural obtenido a partir de la Tuna siguiendo las normas NTE INEN 518, 520, 522, 523, 1670 y los carbohidratos totales por diferencia.

\section{Estudio de coagulación}

\section{Preparación soluciones de referencia}

Se prepararon soluciones de coagulante natural al 1\%, 1,5\% y 2\%. Para la solución al $1 \%$ se pesó $1 \mathrm{~g}$ de coagulante, se introdujo en un matraz aforado y se completó con agua destilada hasta $100 \mathrm{ml}$ y se agita hasta homogenización. Se deja reposar por una hora y se filtra para eliminar cualquier resto de fibra. De forma similar se preparan las soluciones al 1,5\% y $2 \%$. Las soluciones deben usarse de inmediato para evitar su descomposición. 


\section{Pruebas de jarra}

Los ensayos de pruebas de jarra se realizaron en vasos de precipitación de $1000 \mathrm{ml}$.

Para cada una de las pruebas se realizó lo siguiente: Se colocan $1000 \mathrm{ml}$ de agua residual láctea en los vasos de precipitación. Se dosifican con volúmenes de 5, 10, 15, 20, 25, 30 y $35 \mathrm{ml}$ de las soluciones preparadas. Se procede a una mezcla rápida durante $5 \mathrm{~min}$ a $90 \mathrm{rpm}$ y luego una mezcla lenta durante $10 \mathrm{~min}$ a $20 \mathrm{rpm}$. Se deja en reposo 30 min. Se mide la turbidez del sobrenadante de los recipientes de las pruebas de jarra, con un turbidímetro HACH $2100 \mathrm{Q}$ y el color con un espectrofotómetro HACH 2800. Cada prueba se llevó a cabo por duplicado.

\section{Caracterización del agua residual tratada}

Una vez obtenida la dosificación óptima en la prueba de jarras se procede a la caracterización del agua residual tratada. Los parámetros medidos fueron: $\mathrm{pH}$, conductividad, color, turbidez, DQO, DBO, SS, ST, y CF, de acuerdo a los métodos estandarizados (Rice y col., 2017). Los equipos utilizados fueron: un medidor multiparámetro Consort C562, un digestor DQO VELP Scientifica ECO 16

Thermoreactor, un digestor de DBO HACH BODTrack ${ }^{\mathrm{TM}}$ II.

\section{Resultados y Discusión.}

La caracterización de la materia prima (pencas de Tuna) se presenta en la Tabla 2. Los valores de $\mathrm{pH}$ y humedad encontrados son del mismo orden de magnitud de los reportados por Villabona y col. (2013). El calcio y el hierro presentan diferencias mayores. El contenido de metales depende en mayor proporción en la disponibilidad de nutrientes que pueda suministrar el suelo.

TABLA 2: Caracterización de la materia prima

\begin{tabular}{lccc}
\hline PARAMETRO & METODO & RESULTADO & $\begin{array}{l}\text { Villabona y col. } \\
(\mathbf{2 0 1 3})\end{array}$ \\
\hline pH & Potenciométro & 4,53 & 4,42 \\
Humedad(\%) & Gravimétrico & 93,95 & 90,23 \\
Calcio (\%) & $\begin{array}{c}\text { Absorción } \\
\text { Atómica }\end{array}$ & 0,0092 & 0,27 \\
Hierro (\%) & $\begin{array}{c}\text { Absorción } \\
\text { Atómica }\end{array}$ & 0,00078 & 0,00326 \\
\hline
\end{tabular}

Fuente: Grupo Investigación Facultad Ciencias, 2018

El valor de humedad elevado indica que la eficiencia en el polvo de coagulante natural será baja. La mayor energía se consumirá en la eliminación del agua.

Los balances de masa para cada una de las etapas y su rendimiento se muestran en la Tabla 3. 
TABLA 3: Balances de masa por operación

\begin{tabular}{lcccl}
\hline & $\begin{array}{c}\text { Masa de } \\
\text { entrada g }\end{array}$ & $\begin{array}{c}\text { Masa } \\
\text { utilizable g }\end{array}$ & Pérdida g & $\begin{array}{c}\text { Rendim. } \\
\%\end{array}$ \\
\hline $\begin{array}{l}\text { Lavado y pelado } \\
\text { Cortado de }\end{array}$ & 13902,21 & 4886,71 & 9015,5 & 35,2 \\
pulpa & 4886,71 & 4581,48 & 80,65 & 93,8 \\
Secado de pulpa & 4581,48 & 215,12 & 4366,36 & 4,7 \\
Molienda & 215,12 & 204,67 & 10,45 & 95,1 \\
Tamizado & 204,67 & 147,9 & 56,77 & 72,3 \\
Extracción & 147,9 & 133,35 & 14,55 & 90,2 \\
Global & 13902,21 & 133,35 & 13768,86 & 0,96 \\
\hline
\end{tabular}

Fuente: Grupo Investigación Facultad Ciencias, 2018

Los resultados indican que por cada kilogramo de materia prima se obtienen 96 gramos de polvo coagulante natural. Aunque el rendimiento es bajo, las dosis utilizadas en tratamiento de aguas suelen estar en el orden de losppm.

En la Tabla 4 se muestran los resultados de la composición del polvo coagulante.

TABLA 4: Composición del polvo coagulante

\begin{tabular}{lc}
\hline \multicolumn{1}{c}{ Parámetro } & Resultados $(\%)$ \\
\hline Humedad & 5,75 \\
Cenizas & 15,36 \\
Proteínas & 11,60 \\
Fibras & 13,86 \\
Grasas & 1,28 \\
Carbohidratos & 52,15
\end{tabular}

Fuente: Grupo Investigación Facultad Ciencias, 2018

La composición del coagulante sirve para confirmar la capacidad coadyuvante del mismo. Los carbohidratos de alto peso molecular como el almidón, son los principales responsables de dichas propiedades. Así pues, el alto contenido de carbohidratos indica la presencia de polisacáridos que tienen propiedades coagulantes.

Para evaluar la concentración óptima se realizó un test de pruebas de jarra, para lo cual se añadieron diferentes volúmenes $(5,10,15,20,25,30$ y $35 \mathrm{ml})$ de solución de coagulante $(1 \%, 1,5 \%$ y $2 \%)$ al agua residual. Se midieron la turbidez y el color y se calculó la remoción respecto a los valores mostrados en la Tabla 1. En las figuras 1 y 2 se muestran los resultados de remoción.

De los resultados obtenidos el mayor porcentaje de remoción de la turbidez se obtiene al realizar el test de jarras con el $2 \%$ de coagulante natural, ya que se pudo apreciar una remoción de turbidez media de $76,9 \%$. 
FIGURA 1. Porcentaje de remoción de la turbidez a diferentes concentraciones de

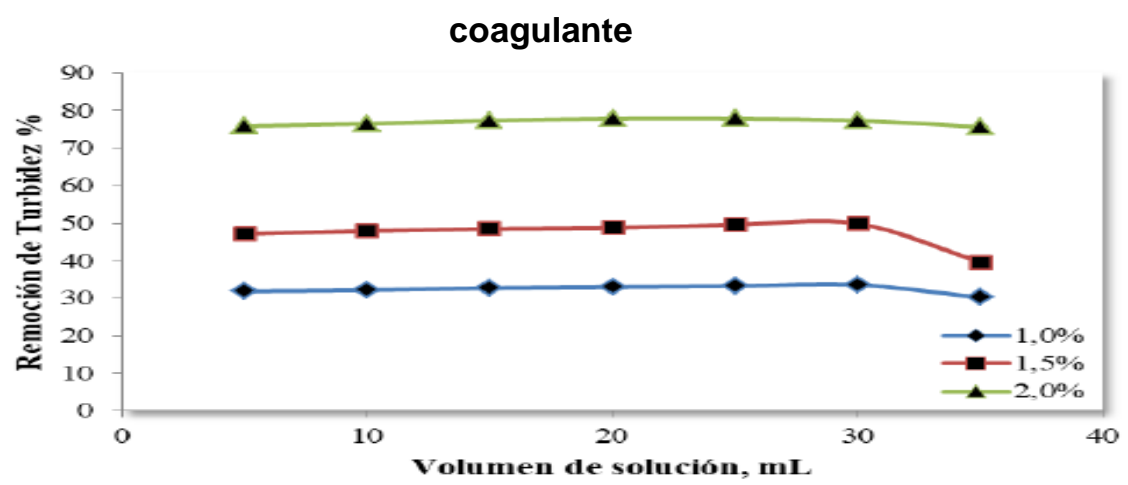

Fuente: Grupo Investigación Facultad Ciencias, 2018

FIGURA 2. Porcentaje de remoción del color a diferentes concentraciones de coagulante natural.

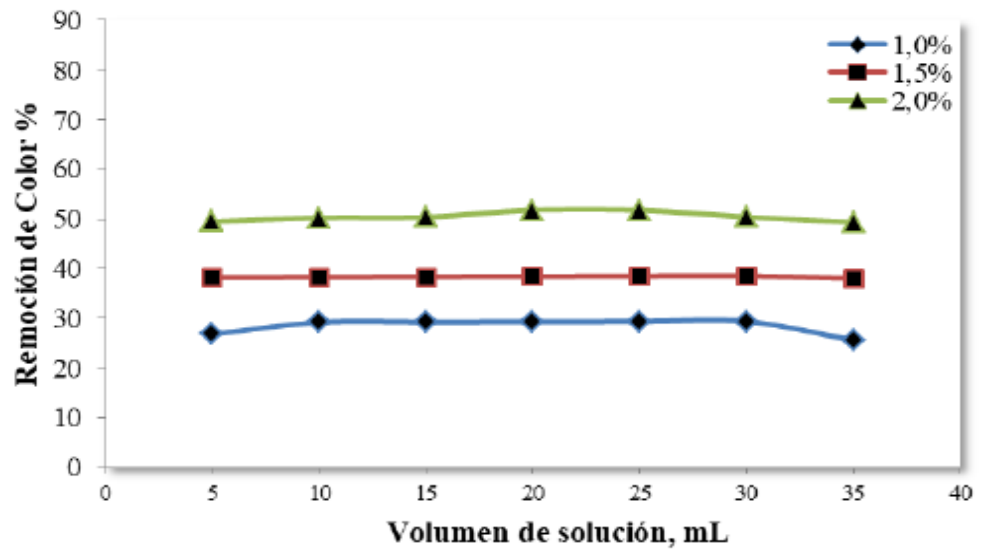

Fuente: Grupo Investigación Facultad Ciencias, 2018

Por otro lado, los resultados obtenidos enseñan que para la prueba de color la mejor dosificación es la de $2 \%$, ya que se apreció una disminución del color en una tasa media de $50,5 \%$, lo cual es indicativo de la capacidad que tiene el coagulante para eliminar los sólidos disueltos y totales que se encuentran en el agua residual.

TABLA 5: Remoción de Turbidez y Color para la concentración de 2\%

\begin{tabular}{lccc}
\hline $\begin{array}{c}\text { Concentración de } \\
\text { Coagulante, } \\
\%\end{array}$ & $\begin{array}{c}\text { Volumen, } \\
(\mathbf{m L})\end{array}$ & $\begin{array}{c}\text { Porcentaje de } \\
\text { remoción de } \\
\text { Turbidez, \% }\end{array}$ & $\begin{array}{c}\text { Porcentaje de } \\
\text { Remoción de } \\
\text { Color, \% }\end{array}$ \\
\hline & 5 & 75,9 & 49,49 \\
& 10 & 76,45 & 50,26 \\
$2,0 \%$ & 15 & 77,29 & 50,38 \\
& 20 & 77,84 & 51,79 \\
& 25 & 77,84 & 51,77 \\
Media & 30 & 77,29 & 50,41 \\
Desviación & 35 & 75,62 & 49,36 \\
\hline
\end{tabular}

Fuente: Grupo Investigación Facultad Ciencias, 2018 
El volumen óptimo de solución de coagulante al 2\% fue de $20 \mathrm{ml}$ (400 ppm), para una remoción de turbidez de 77,84\% y de color de 51,79\% (Ver Tabla 5).

Una vez establecida la dosificación óptima se procedió a evaluarlos demás parámetros incluidos en la caracterización como se pueden observar en la Tabla 6.

TABLA 6: Caracterización del agua residual tratada

\begin{tabular}{lcccc}
\hline Parámetro & Unidades & Método* $^{*}$ & Valor & TULSMA \\
\hline pH & Und. & $4500-\mathrm{B}$ & 5,32 & $6-9$ \\
Conductividad & $\mu$ Siems/cm & $2510-\mathrm{B}$ & 980 & - \\
Color & Und. Co/Pt & - & 1880 & - \\
Turbiedad & NTU & $2130-\mathrm{B}$ & 80 & - \\
DBO & $\mathrm{mg} / \mathrm{L}$ & $5220-\mathrm{C}$ & 4230 & 200 \\
DQO & $\mathrm{mg} / \mathrm{L}$ & $5210-\mathrm{B}$ & 2120 & 100 \\
SS & $\mathrm{mg} / \mathrm{L}$ & $2540-\mathrm{D}$ & 1052 & 130 \\
ST & $\mathrm{mg} / \mathrm{L}$ & $2540-\mathrm{A}$ & 5400 & 1600 \\
CF & UFC/100 mL & Microfiltr & $<1000$ & 2000 \\
\hline *Métodos Normalizados & \multicolumn{2}{c}{} \\
\multicolumn{2}{r}{ Fuente: Grupo Investigación Facultad Ciencias, 2018 }
\end{tabular}

Las eficiencias de remoción encontradas en los otros parámetros analizados fueron: $26,8 \%$ en DQO, $13,9 \%$ en DBO, $31,0 \%$ en SS, $26,4 \%$ en ST y 99,96\% en CF. Debe resaltarse el poder bactericida de la tuna al obtenerse un valor tan elevado en la remoción de coliformes fecales. También debe resaltarse, que aun cuando se lograron reducciones importantes en los parámetros seleccionados, todavía no se cumple con la normativa ecuatoriana TULSMA, excepto por los coliformes fecales.

\section{Conclusiones.}

- El rendimiento encontrado en coagulante natural a partir de las pencas de tuna fue de $0,96 \%$.

- La eficiencia de un coagulante puede llevarse a cabo mediante la medición de la turbidez y el color para diferentes concentraciones aplicadas. La viabilidad final de la aplicación del coagulante será de acuerdo a una relación eficiencia y costo de producción del coagulante.

- Las eficiencias de remoción más resaltantes usando la Tuna fueron: turbidez $77,84 \%$, color $51,79 \%$ y $99,96 \%$ en coliformes fecales.

- Se puede utilizar el polvo de coagulante como tratamiento preliminar de las aguas residuales de una industria láctea en Ecuador.

\section{Referencias bibliográficas.}

Barka N, Ouzaouit K, Abdennouri M, El Makhfouk M, (2013). Dried prickly pear cactus (Opuntia ficus indica) cladodes as a low-cost and eco-friendly 
biosorbent for dyes removal from aqueous solutions, Journal of the Taiwan Institute of Chemical Engineers, 44, pp. 52-60.

Buntner D, Sánchez A, Garrido JM, (2013). Feasibility of combined UASB and MBR system in dairy wastewater treatment at ambient temperatures, Chemical Engineering Journal, Vol. 230, pp. 475-481.

Chen W, Liu J, (2012). The possibility and applicability of coagulation-MBR hybrid system in reclamation of dairy wastewater, Desalination, 285, pp. 226-231.

Choudhary M, Ray MB, Neogi S, (2019). Evaluation of the Potential Application of Cactus (Opuntia ficus-indica) as a Bio-coagulant for Pre-treatment of Oil Sands Process- Affected Water, Separation and Purification Technology, 209, pp. 714-724.

Diaz A, Rincon N, Escorihuela A, Fernandez N , Chacin E, Forster CF, (1999). A preliminary evaluation of turbidity removal by natural coagulants indigenous to Venezuela, Process Biochemestry, 35, pp. 391-395.

Djerroud N, Adjeroud N, Felkai-Haddache L, Hammoui Y, Remini H, Dahmoune F, Merzouk B, Madani K, (2018). Enhanced electrocoagulation-electroflotation for turbidity removal by Opuntia ficus indica cladode mucilage, Water and Environmental Journal, Vol. 32, 3, pp. 321-332.

El-Mostafa K, El Kharrassi Y, Badreddine A, Andreoletti P, Vamecq J, El Kebbaj M, Latruffe N, Lizard G, Nasser B, Cherkaoui-Malki M, (2014). Nopal Cactus (Opuntia ficus- indica) as a Source of Bioactive Compounds for Nutrition, Health and Disease, Molecules, 19, 14879-14901.

Formentini-Schmitt DM, Dias AC, Veit MT, Bergamasco R, Salcedo AM, FagundesKlen MR, (2013). Ultrafiltration Combinedwith Coagulation / Flocculation / Sedimentation using Moringa oleifera as Coagulant to Treat Dairy Industry Wastewater, Water Air Soil Pollut, 224, pp. 1682-1691.

Gade A, Gaikwad S, Tiwari V, Yadav A, Ingle A, Rai M, (2010). Biofabrication of Silver Nanoparticles by Opuntia ficus-indica: In vitro Antibacterial Activity and Study of the Mechanism Involved in the Synthesis, Current Nanoscience, 6, 4, pp. 370-375.

Geetha M, Dumaran JJ, Feroz S, (2012). Dairy Wastewater Treatment Using Low Molecular Weight Crab Shell chitosan, Journalof The Institution of Engineers (India) Series E, 93, 1, pp. 9-14.

Gomes L, Troiani EP, Malpass G, Jorge Nozaki J, (2016). Opuntia ficus indica as a polyelectrolyte source for the treatment of tannery wastewater, Desalination and Water Treatment, 57, 22, 10181-10187. 
Kaewsuk J, Thorasampan W, Thanuttamavong M, Seo GT, (2010). bKinetic development and evaluation of membrane sequencing batch reactor (MSBR) with mixed cultures photosynthetic bacteria for dairy wastewater treatment, Journal of Environmental Management, 91, pp. 1161-1168.

Karadag D, Oguz Emre Köroğlu OE, Ozkaya B, Cakmakci M, (2015). A review on anaerobic biofilm reactors for the treatment ofdairy industry wastewater, Process Biochemistry, 50, 2, pp. 262-271.

Kushwaha JP, Srivastava VC, Mall ID, (2010). Treatment of dairy wastewater by commercial activated carbon and bagasse fly ash: Parametric, kinetic and equilibrium modelling, disposal studies, Bioresource Technology, 101, pp. 3474-3483.

Kushwaha JP, Srivastava VC, Mall ID, (2010). Treatment of dairy wastewater by inorganic coagulants: Parametric and disposal studies, Water Research, 44, pp. $5867-5874$.

Kushwaha JP, Srivastava VC, Mall ID, (2011). Studies on Electrochemical Treatment of Dairy Wastewater Using Aluminum Electrode, American Institute of Chemical Engineers Journal, 57, 2, pp. 2589-2598.

Louati I, Fersi M, Hadrich B, Ghariani B, Nasri M, Mechichi T, (2018). Prickly pear cactus cladodes powder of Opuntia ficus indica as a cost effective biosorbent for dyes removal from aqueous solutions, Biotech, 8, pp. 477-486.

Lozoya Castillo DL, Castillo-Hernández SL, Hernández- Marín DA, Rivas-Morales C, Sánchez-García E, (2018). Evaluación de la actividad antimicrobiana, antiinflamatoria y antioxidante de subproductos de Opuntia ficus-indica y Mangifera indica, Investigación y Desarrollo en Ciencia y Tecnología de Alimentos, 3, pp. 139-144.

Luo J, Ding LH, 2011, Influence of $\mathrm{pH}$ on treatment of dairy wastewater by nanofiltration using shear-enhanced filtration system, Desalination, 278, pp. 150-156.

NTE INEN 518, (1981). Harinas de origen vegetal. Determinación de la pérdida por calentamiento Norma Técnica Ecuatoriana, Primera edición. Disponible en: http://apps.normalizacion.gob.ec/descarga/ $\quad(2019,1$ de Abril).

NTE INEN 520, (2013). Harinas de origen vegetal.. Determinación de ceniza.. Norma Técnica Ecuatoriana, Primera edición. Disponible en: http://apps.normalizacion.gob.ec/descarga/, (2019, 1 de Abril). 
NTE INEN 522, 2013, Harinas de origen vegetal. Determinación de la fibra cruda. Norma Técnica Ecuatoriana. Primera edición. Disponible en: http://apps.normalizacion.gob.ec/ descarga/ , (2019, 1 de Abril).

NTE INEN 523, 1981, Harinas de origen vegetal. Determinación de la grasa. Norma Técnica Ecuatoriana, Primera edición. Disponible en: http://apps.normalizacion.gob.ec/descarga/ (2019, 1 de Abril).

NTE INEN 1670, 1988, QUINUA. Determinación de la proteína total (proteína cruda). Norma Técnica Ecuatoriana, Primera edición. Disponible en: http://apps.normalizacion.gob.ec/descarga/ (2019, 1 de Abril).

Onditi M, Adelodun A, Changamu EO, Ngila JC, (2016). Removal of Pb2 and Cd2 from drinking water using polysaccharide extract isolated from cactus pads (Opuntia ficus indica), Journal of Applied Polymer Science, Vol. 133, 38, pp. 43913-43911.

Sarkar B, Chakrabarti PP, Vijaykumar A., Kale V, (2006) Wastewater treatment in dairy industries -possibility of reuse, Desalination, 195, pp.141-152.

Selmer-Olsen E, Ratnaweera HC, Pehrsonv R, (1996). A novel treatment process for Dairy wastewater with chitosan produced from shrimp-shell waste, Water Science and Technology, 34, 11, pp. 33-40.

TULSMA, (2015). Ministerio del Ambiente. Consultado el 10 de enero de 2019 de:......http://www.industrias.ec/archivos/CIG/file/CARTELERA/Refor ma\%20Anexo\%2028\%20feb\%202014\%20FINAL.pdf.

Villabona A, Paz IC, Martínez J, (2013). Caracterización de la Opuntia ficus-indica para su uso como coagulante natural, Revista Colombiana de Biotecnología, $\mathrm{XV}, 1,137-144$.

Vourch M, Balannec B, Chaufer B, Dorange G, (2008) Treatment of dairy industry wastewater by reverse osmosis for water reuse, Desalination, 219, pp. 190-202.

Wan J, Chakraborty T, Xu C, Ray MB, (2019). Treatment train for tailings pond water using Opuntia ficus-indica as coagulant, Separation and Purification Technology, Vol. 211, pp. 448-455.

Yavuz Y, Ocal E, Koparal AS, Ogutveren UB, (2011). Treatment of dairy industry wastewater by $\mathrm{EC}$ and $\mathrm{EF}$ processes using hybrid $\mathrm{Fe}-\mathrm{Al}$ plate electrodes, Journal of Chemical Technology \& Biotechnology, 86, pp. 964-969.

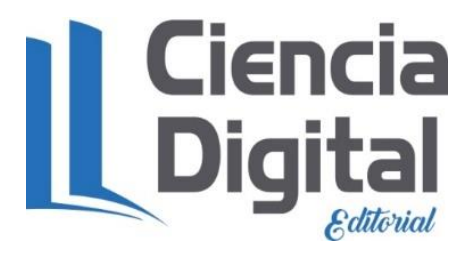




\section{PARA CITAR EL ARTÍCULO INDEXADO.}

Borja Mayorga, D. F., Chuiza Rojas, M. R., \& Andrade Avalos, M. L. (2021). Clarificación de aguas residuales de la industria láctea en base a la tuna (Opuntia ficus indica) como coagulante natural. ConcienciaDigital, 4(3.1), 177-189. https://doi.org/10.33262/concienciadigital.v4i3.1.1821

\section{¿Ciencia}

El artículo que se publica es de exclusiva responsabilidad de los autores y no necesariamente reflejan el pensamiento de la Revista Conciencia Digital.

El artículo queda en propiedad de la revista y, por tanto, su publicación parcial y/o total en otro medio tiene que ser autorizado por el director de la Revista Conciencia Digital.

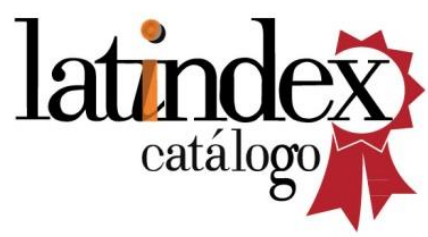

